\title{
Real-time, wide-field, and quantitative oxygenation imaging using spatiotemporal modulation of light
}

\author{
Manon Schmidt \\ Enagnon Aguénounon \\ Amir Nahas \\ Murielle Torregrossa \\ Bruce J. Tromberg \\ Wilfried Uhring \\ Sylvain Gioux
}




\title{
Real-time, wide-field, and quantitative oxygenation imaging using spatiotemporal modulation of light
}

\author{
Manon Schmidt, ${ }^{a}$ Enagnon Aguénounon, ${ }^{a}$ Amir Nahas, ${ }^{a}$ Murielle Torregrossa, ${ }^{a}$ Bruce J. Tromberg, ${ }^{b, c}$ \\ Wilfried Uhring, ${ }^{a}$ and Sylvain Gioux ${ }^{\mathrm{a}, *}$ \\ aUniversity of Strasbourg, ICube Laboratory, Strasbourg, France \\ ${ }^{\mathrm{b}}$ Beckman Laser Institute and Medical Clinic, Laser Microbeam and Medical Program, Irvine, California, United States \\ 'University of California, Department of Biomedical Engineering, Irvine, California, United States
}

\begin{abstract}
Quantitative diffuse optical imaging has the potential to provide valuable functional information about tissue status, such as oxygen saturation or blood content to healthcare practitioners in real time. However, significant technical challenges have so far prevented such tools from being deployed in the clinic. Toward achieving this goal, prior research introduced methods based on spatial frequency domain imaging (SFDI) that allow real-time (within milliseconds) wide-field imaging of optical properties but at a single wavelength. However, for this technology to be useful to clinicians, images must be displayed in terms of metrics related to the physiological state of the tissue, hence interpretable to guide decision-making. For this purpose, recent developments introduced multispectral SFDI methods for rapid imaging of oxygenation parameters up to 16 frames per seconds (fps). We introduce real-time, wide-field, and quantitative blood parameters imaging using spatiotemporal modulation of light. Using this method, we are able to quantitatively obtain optical properties at $100 \mathrm{fps}$ at two wavelengths (665 and $860 \mathrm{~nm}$ ), and therefore oxygenation, oxyhemoglobin, and deoxyhemoglobin, using a single camera with, at most, $4.2 \%$ error in comparison with standard SFDI acquisitions. ๑ The Authors. Published by SPIE under a Creative Commons Attribution 4.0 Unported License. Distribution or reproduction of this work in whole or in part requires full attribution of the original publication, including its DOI. [DOI: 10.1117/1.JBO.24.7.071610]
\end{abstract}

Keywords: oxygenation imaging; diffuse optical imaging; spatial frequency domain imaging.

Paper 180634SSR received Nov. 23, 2018; accepted for publication Jan. 28, 2019; published online Mar. 13, 2019.

\section{Introduction}

Diffuse optical imaging using either temporal or spatial modulation of light has shown strong promise for quantitatively assessing functional parameters in living tissue, such as content in oxyhemoglobin, deoxyhemoglobin, water, and lipids. Such parameters can be used to determine the physiological condition of the tissue and provide critical information to healthcare professionals for decision making. For instance, temporal frequency domain monitoring of breast tissue during neoadjuvant chemotherapy treatment can help differentiating responding versus nonresponding patients within 10 days of treatment. ${ }^{1,2}$ In other studies, spatial frequency domain imaging (SFDI) has demonstrated the potential to diagnose patients early with diabetic foot ulcers, providing means to adapt treatment toward limiting morbidity and decreasing healthcare burden. ${ }^{3,4}$

While these methods have demonstrated a strong potential to aid in decision making and solve concrete clinical problems, their adaptation to challenging clinical environments has been very limited. Surgery is one of these environments where information must be provided quantitatively (i.e., repeatable and interpretable), in real time (i.e., $>25$ images per seconds), and over a large field-of-view (i.e., $>10 \times 10 \mathrm{~cm}^{2}$ ). ${ }^{5}$ To attain this challenging objective, our group has been developing solutions based on SFDI. Because SFDI works in the frequency domain, it allows the capture of information for all the pixels of an image at once, providing a significant advantage over raster-scanning methods. The original developments of SFDI relied on the acquisition of several images at single

\footnotetext{
*Address all correspondence to Sylvain Gioux, E-mail: sgioux@ unistra.fr
}

wavelengths, providing quantitative multipixel optical properties measurements. ${ }^{6-8}$ More recent developments have introduced near-real-time [e.g., 10 frames per second (fps)] and real-time (i.e., $>25 \mathrm{fps}$ ) imaging methods such as single snapshot of optical properties (SSOP). ${ }^{9-11}$ These methods process the acquired information directly in the frequency domain offering the opportunity to drastically reduce the acquisition time down to a single acquired image for obtaining optical properties maps of living tissues. However, these methods still acquire only one wavelength at a time, making them suboptimal for imaging living tissue parameters (e.g., chromophores concentration) in real time.

In order to deploy such technology in the operating room, methods capable of performing multi- or hyperspectral imaging quantitatively, over a large field-of-view and in real time, must be developed. Recent work has proposed dual-wavelengths imaging for extracting blood parameters such as the concentration of oxyhemoglobin, deoxyhemoglobin, or oxygenation. For instance, SSOP was used in conjunction with speckle imaging using a scientific CMOS camera by sequentially acquiring wavelengths, leading to $16 \mathrm{fps}$ imaging. ${ }^{12}$ However, this work uses a mechanical optical chopper and low-power $(150 \mathrm{~mW})$ laser diodes, making it difficult to achieve higher frame rates and limiting its scalability to a higher number of wavelengths. Another, more recent work used square patterns to improve switching time using a standard digital micromirror device (DMD) to project three wavelengths sequentially at three phases (standard SFDI acquisition), and compressed single pixel imaging leading to $12.5 \mathrm{fps}$ imaging. ${ }^{13}$ However, relying on sequential frames to acquire optical properties maps naturally restricts the possibility to achieve higher frame rates. As of today, because of these instrumental limitations, no method has been 
developed for real-time (i.e., $>25$ images per seconds), widefield (i.e., $>10 \times 10 \mathrm{~cm}^{2}$ ), and quantitative imaging for clinical applications.

In this work, we present a method using both spatial and temporal modulation of light to provide images interpretable by clinicians in time-constrained environments such as surgery. We propose here a method where sources are modulated sinusoidally in time using signal generators (no mechanical chopper) and projected in the form of single-frame patterns for processing using the SSOP method (no need for sequential frames). Sources are modulated in time to encode wavelengths and combined in a multifiber bundle connected to a spatial-light modulator. Light is then projected with specific patterns onto the field-of-view and images captured using a fast camera. The obtained threedimensional (3-D) cube of data (two-dimensions + time) is then processed in the temporal frequency domain to separate wavelengths, and the resulting images are processed in the spatial frequency domain using the SSOP methodology to extract optical properties for each wavelength and then calculate blood parameters (oxyhemoglobin, deoxyhemoglobin, and oxygenation). In the first part of this paper, we present the imaging method, processing, and hardware. Then, we validate the method in comparison with standard, non-real-time SFDI onto tissue-mimicking phantoms. Finally, we perform a validation on a living hand during an arm-cuff occlusion. Altogether, this study contributes to the translation of endogenous diffuse optical imaging for surgical guidance.

\section{Material and Methods}

\subsection{Spatiotemporal Modulation of Light}

The concept of multispectral imaging using temporal modulation of light sources for wavelength encoding is not new, nor are multispectral spatial frequency domain methods. ${ }^{13-18}$ However, until now, imaging living tissue parameters over large fields-of-view at high resolution, quantitatively, and in real time remained a significant challenge. The principle underlying the spatiotemporal modulation of light method is shown in Fig. 1. Discrete sources are modulated in time, each wavelength being at a different specifically chosen temporal frequency. The light outputs from all sources are combined using a multifiber bundle connected to a spatial light modulator. Patterns of light combining all temporally modulated wavelengths are projected onto the field-of-view and a fast camera is used to acquire the scene. The acquired images are first processed using a temporal discrete Fourier transform (DFT) to accurately separate wavelengths contributions. Finally, the resulting images are processed in the spatial frequency domain using SSOP methodology to obtain the optical properties at the chosen wavelengths.

One of the significant hurdles in implementing such method is in ensuring that wavelengths are precisely and correctly modulated, sampled, and processed to unambiguously extract the information content contained in each temporally modulated source. Not performing these operations correctly would result in inaccuracies in the extracted optical properties and therefore in the assessment of tissue parameters. Two strategies can be used to perform these measurements: either a fine temporal sampling of the scene allowing to use a generic fast Fourier transform (FFT) function to separate any temporal frequency contribution, or a custom DFT function allowing to sample only a chosen number of frequencies. While a generic FFT may be more suited to analyze a large amount of contributions, it can be more costly in terms of acquisition and processing time compared with a custom DFT. In this work, because we focus on acquiring real-time images, we chose to implement a custom DFT to allow ultrafast computation of the chosen sources' contributions. The DFT of one pixel in time is given as

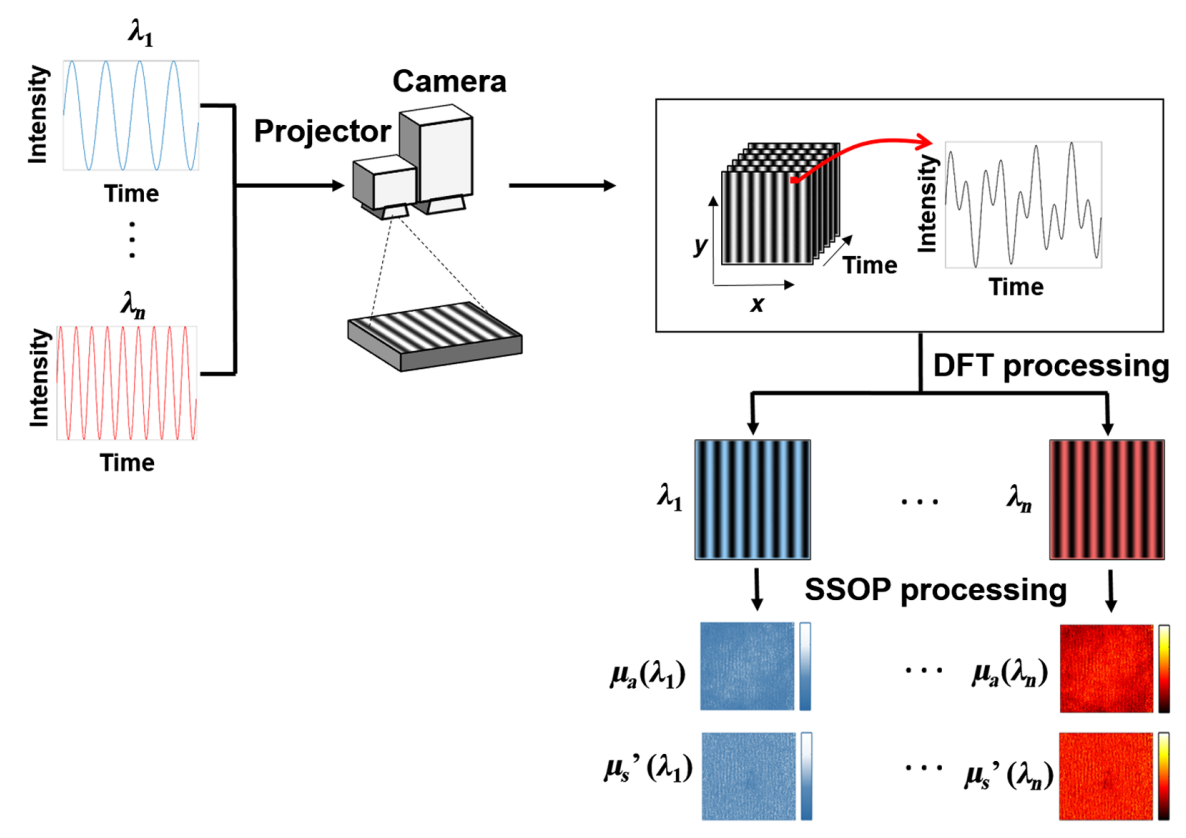

Fig. 1 Spatiotemporal modulation of light: acquisition and processing. Sources at different wavelengths are modulated in time at specifically chosen frequency. Light from these sources is combined and modulated in space using a spatial light modulator. The scene is acquired using a fast camera. The acquired images are then processed in the temporal frequency domain to separate the contribution from the different light sources, and processed in the spatial frequency domain to extract the optical properties at all wavelengths. 


$$
X(k)=\sum_{n=1}^{N} x(n) \cdot e^{-\frac{j 2 \pi k(n-1)}{N}},
$$

and the proper frequencies that should be used to modulate are exact frequencies in the discrete Fourier domain, for instance, in the case of four sources:

$f_{1}=\frac{k_{1} \cdot F_{e}}{N} ; \quad f_{2}=\frac{k_{2} \cdot F_{e}}{N} ; \quad f_{3}=\frac{k_{3} \cdot F_{e}}{N} ; \quad f_{4}=\frac{k_{4} \cdot F_{e}}{N}$,

with $F_{e}$ being the sampling frequency, $N$ is the number of acquired images, and $k_{i}$ determined such that the captured signal spans an integer number of periods. ${ }^{19,20}$

If these conditions are gathered, on top of obvious considerations such as avoiding harmonics or standard electrical current frequencies, and along with a precise synchronization of the camera and signal generators, the contribution of each source can be precisely and unambiguously determined.

\subsection{Acquisition and Processing}

In this work, we performed acquisitions at a sampling frequency $\mathrm{Fe}=100 \mathrm{~Hz}$, processing $N=10$ images, and modulated two wavelengths to determine oxygenation, $\lambda_{1}=665 \mathrm{~nm}$ with $k_{1}=$ $3\left(f_{1}=30 \mathrm{~Hz}\right)$ and $\lambda_{2}=860 \mathrm{~nm}$ with $k_{2}=2\left(f_{2}=20 \mathrm{~Hz}\right){ }^{21}$ The number of images $(N=10)$ was chosen such that all parameters have been adjusted to satisfy the conditions for proper DFT processing (Sec. 2.1) while maintaining a proper signal-to-noise ratio (SNR) in the acquired images. We implemented a custom rolling window algorithm of the DFT method onto Matlab with the parallel computing toolbox (using a graphics processing unit, GPU) by adding the $N+1$ term of the DFT sum and subtracting the first term of the DFT sum in real time. More details regarding the GPU implementation of the processing method are available elsewhere. ${ }^{20}$

All acquisitions have been performed on the imaging system described in the next section. In order to handle the large flux of data, as well as control the hardware and perform GPU processing of the acquired images, a personal computer with the following characteristics was used: Intel i7-7800x $3.5 \mathrm{GHz}$ central processing unit, $16 \mathrm{~GB}$ of RAM, four $1 \mathrm{~TB}$ solid-state drives for data acquisition and one $500 \mathrm{~GB}$ solid-state drive for system operation, and an NVIDIA GeForce GTX1080TI GPU.

\subsection{Imaging System}

The instrumental setup was custom built using a DMD (Vialux, Germany) for the projection of custom patterns, fiber-coupled to two laser diodes at 665 and $860 \mathrm{~nm}$ (LDX Optronics, Maryville, Tennessee). Diodes were mounted in a temperature-controlled mount (TCLDM9, Thorlabs, Newton, New Jersey). Intensity from the diodes was controlled using current controllers (TDC240C, Thorlabs, Newton, New Jersey) and temperature controlled using thermoelectric cooler controllers (TED200C, Thorlabs, Newton, New Jersey). Diodes were modulated using a dedicated 20-MHz dual channel function generator (4047B, BK Precision, Yorba Linda, California). The laser diodes were coupled into a 2-to-1 fiber bundle (BFY1000HS02, Thorlabs, Newton, New Jersey) connected to the DMD. The projection
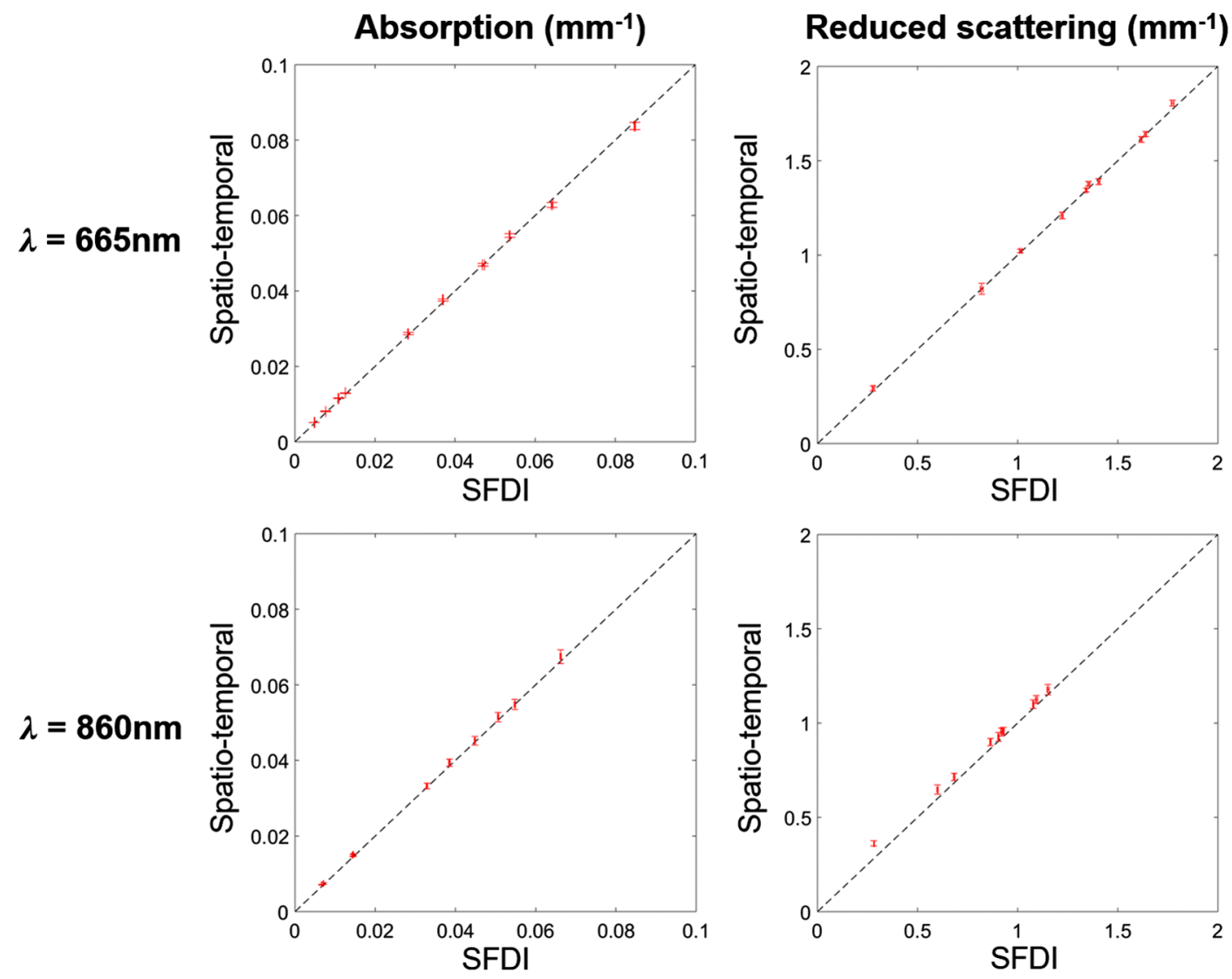

Fig. 2 Results from phantoms experiments. Absorption and reduced scattering are shown (mean and standard deviation) over a wide range of optical properties. Results are shown for both wavelengths using standard SFDI as a reference and spatiotemporal modulation of light. 
system projects a sine wave pattern over a $200 \times 150 \mathrm{~mm}^{2}$ surface at $45-\mathrm{cm}$ working distance. Images were acquired over a $130 \times$ $130 \mathrm{~mm}^{2}$ field-of-view and at a resolution of $1024 \times 1024$ pixels using a scientific-grade monochrome sCMOS camera (PCO Edge 5.5, Kelheim, Germany). The camera and signal generators were synchronized together using a second $20-\mathrm{MHz}$ dual channel function generator as a reference (4047B, BK Precision, Yorba Linda, California). Polarizers (PPL05C, Moxtek, Orem, Utah), arranged in a crossed configuration, were used to minimize the contribution from specular reflections at the surface of the sample. A silicone-based optical phantom was used for calibration and built using titanium dioxide $\left(\mathrm{TiO}_{2}\right)$ as a scattering agent and India ink as an absorbing agent. ${ }^{22}$ Its large size $(210 \mathrm{~mm} \times 210 \mathrm{~mm} \times 20 \mathrm{~mm})$ accommodates the system's field-of-view with reduced scattering $\mu_{s}^{\prime}=1.08 \mathrm{~mm}^{-1}$ and absorption $\mu_{a}=0.012 \mathrm{~mm}^{-1}$ at $665 \mathrm{~nm}$, and $\mu_{s}^{\prime}=$ $0.78 \mathrm{~mm}^{-1}$ and $\mu_{a}=0.022 \mathrm{~mm}^{-1}$ at $860 \mathrm{~nm}$.

\subsection{Validation Experiments}

\subsubsection{Acquisition protocol}

SFDI and spatiotemporal modulation of light experiments were performed sequentially. SFDI was used as a reference by acquiring a total of six images for each wavelength (with no temporal modulation) at spatial frequencies of 0 and $0.3 \mathrm{~mm}^{-1}$ with three evenly spaced phases each to extract the amplitude modulation and the calibrated diffuse reflectance. A lookup table (LUT) generated from Monte-Carlo was used to extract the optical properties for each wavelength following acquisition. Extensive details regarding the SFDI acquisition and LUT processing method can be found elsewhere. ${ }^{7,23,24}$ Spatiotemporal acquisitions were performed at $30 \mathrm{~Hz}$ for $665 \mathrm{~nm}$ and $20 \mathrm{~Hz}$ for $860 \mathrm{~nm}$. Acquisition rate of the camera was set at $100 \mathrm{fps}$. The SSOP projection frequency was $0.3 \mathrm{~mm}^{-1}$ and the acquired data postprocessed, first in time to extract contributions from the sources, and then in space to extract the optical properties for each wavelength. More details regarding the temporal processing can be found in Ref. 20, and details regarding the SSOP acquisition and processing method can be found in Refs. 9 and 11 .

\subsubsection{Phantom experiments}

A set of 10 tissue-mimicking phantoms were built using titanium dioxide $\left(\mathrm{TiO}_{2}\right)$ as a scattering agent and nigrosin as an absorbing agent. ${ }^{25}$ Phantoms were all acquired using both SFDI and spatiotemporal modulation of light, postprocessed,

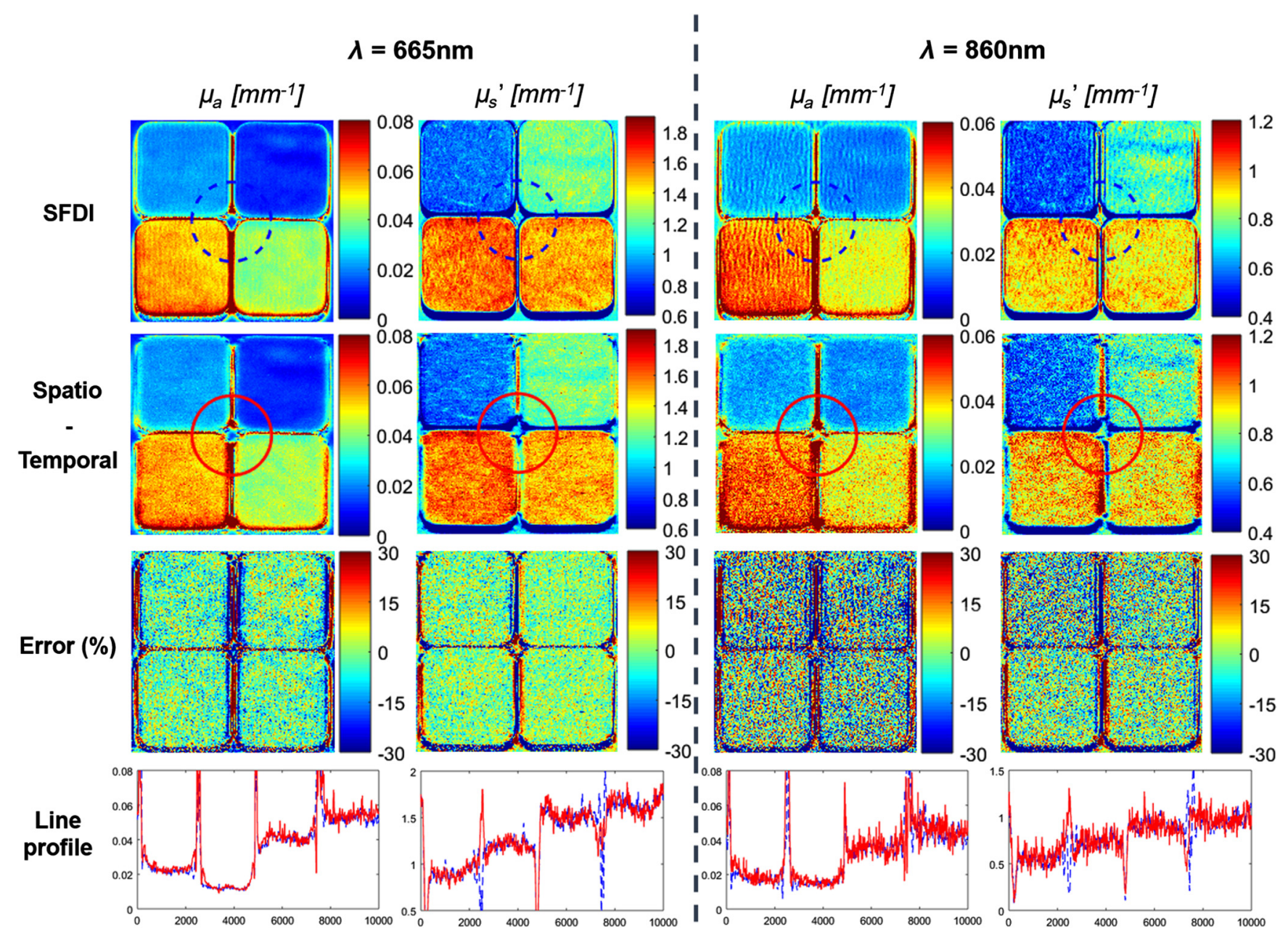

Fig. 3 Actual images of tissue mimicking phantoms. Absorption and reduced scattering images of four phantoms are shown over a range of optical properties. Results are shown for both wavelengths using standard SFDI as a reference and spatiotemporal modulation of light. In addition, relative error maps are shown and line profiles compared (dashed blue: standard SFDI, solid red: spatiotemporal modulation of light). 
and the mean error in percent calculated over $30 \times 30 \mathrm{~mm}^{2}$ region of interest taken at the center of each phantom.

\subsubsection{In vivo experiments}

A human hand was used to illustrate the capacity of the method to work properly during in vivo conditions and in real time. First, a still hand was imaged using SFDI and spatiotemporal modulation of light to compare in vivo results using both methods. Mean error in percent calculated over $50 \times 50 \mathrm{~mm}^{2}$ region of interest taken on the back of the hand. Then, a simple armcuff occlusion experiment was performed during $10 \mathrm{~min}$, with $3 \mathrm{~min}$ of rest, $4 \mathrm{~min}$ of occlusion, and $3 \mathrm{~min}$ of recovery. Images were acquired and postprocessed using the spatiotemporal modulation of light method and the absorption coefficients used to determine oxygen saturation through the calculation of the concentrations of oxy- and deoxyhemoglobin. ${ }^{21}$

\section{Results}

\subsection{Phantom Experiments}

Results from the phantom experiments are summarized in Fig. 2. On average, optical properties obtained using spatiotemporal modulation of light show good agreement with the standard SFDI method. Mean errors were calculated for all the pixels in a $30 \times 30 \mathrm{~mm}^{2}$ region taken in the middle of each phantom. For $665 \mathrm{~nm}$, the mean error is $1.8 \% \pm 1.2 \%$ in absorption and $3.3 \% \pm 1 \%$ in reduced scattering. For $860 \mathrm{~nm}$, the mean error is $4.2 \% \pm 2.1 \%$ in absorption and $2.5 \% \pm 1.1 \%$ in reduced scattering.

Actual images of four phantoms are shown in Fig. 3 for both wavelengths, in absorption and reduced scattering. Results from standard SFDI are shown, as well as spatiotemporal modulation of light, a percentage error map comparing both methods, and a line profile. Overall good visual agreement is confirmed in these images.

\subsection{In Vivo Experiments}

Images from the still hand are shown in Fig. 4 for both wavelengths, in absorption and reduced scattering. Results from standard SFDI are shown, as well as spatiotemporal modulation of light, a percentage error map comparing both methods, and a line profile. Overall good visual agreement is confirmed in these images. Mean errors were calculated for all the pixels in a $50 \times 50 \mathrm{~mm}^{2}$ region on the back of the hand. For $665 \mathrm{~nm}$, the mean error is $1.9 \% \pm 2.0 \%$ in absorption and $2.0 \% \pm 0.7 \%$ in

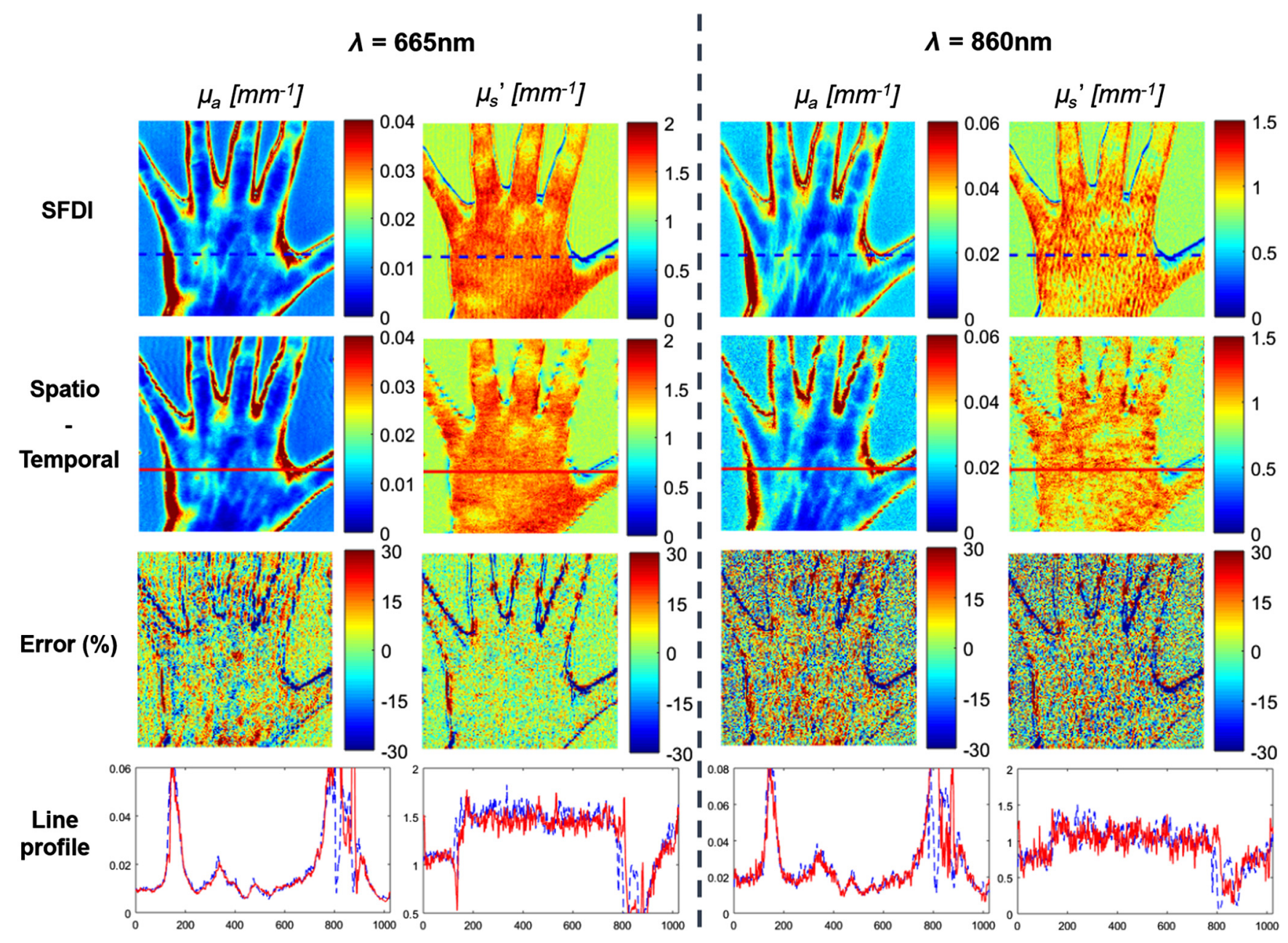

Fig. 4 Actual images of a still hand. Absorption and reduced scattering images of a still hand are shown over a range of optical properties. Results are shown for both wavelengths using standard SFDI as a reference and spatiotemporal modulation of light. In addition, relative error maps are shown and line profiles compared (dashed blue: standard SFDI, solid red: spatiotemporal modulation of light). 

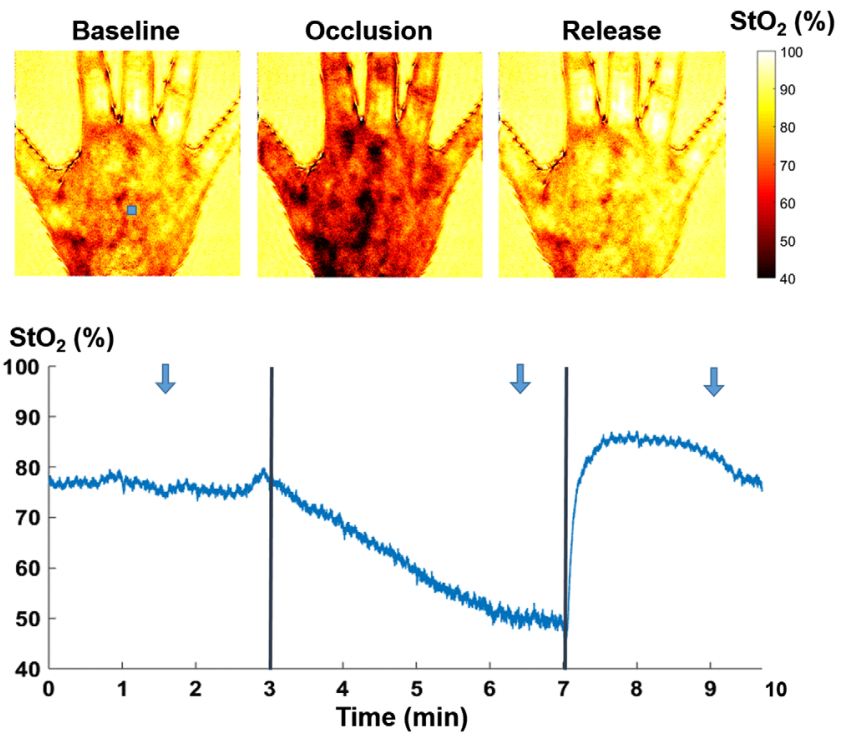

Fig. 5 Arm-cuff occlusion results. An arm-cuff occlusion was performed for $4 \mathrm{~min}$, following $3 \mathrm{~min}$ of resting state, and followed by $3 \mathrm{~min}$ of recovery. Images were acquired using spatiotemporal modulation of light at $100 \mathrm{fps}$ and processed for oxygenation. Top images show oxygen saturation at three time points during the baseline resting measurement, the occlusion, and after the release. The saturation of a point in the hand (blue square) is shown in the plot below. Time points of the above images are indicated by arrows.

reduced scattering. For $860 \mathrm{~nm}$, the mean error is $3.4 \% \pm 1.9 \%$ in absorption and $0.3 \% \pm 1.7 \%$ in reduced scattering. These results confirm the phantom experiments with similar error values on average and allow to appreciate the image quality of the extracted optical properties maps. In particular, the vein structures are clearly visible on the absorption images confirming the potential for the spatiotemporal modulation of light method to perform adequately for clinically relevant use.

Finally, results from the arm-cuff occlusion experiment are shown in Fig 5. Significant decrease in oxygen saturation is visible during the occlusion, with values going from $78 \%$ down to $49 \%$, following by a hyperemia phase after the release of the occlusion with a slow decrease back to the resting state.

A movie is shown in Fig. 6. Note that the movie frame rate has been decreased from 100 to 25 fps to reduce the size of the file.

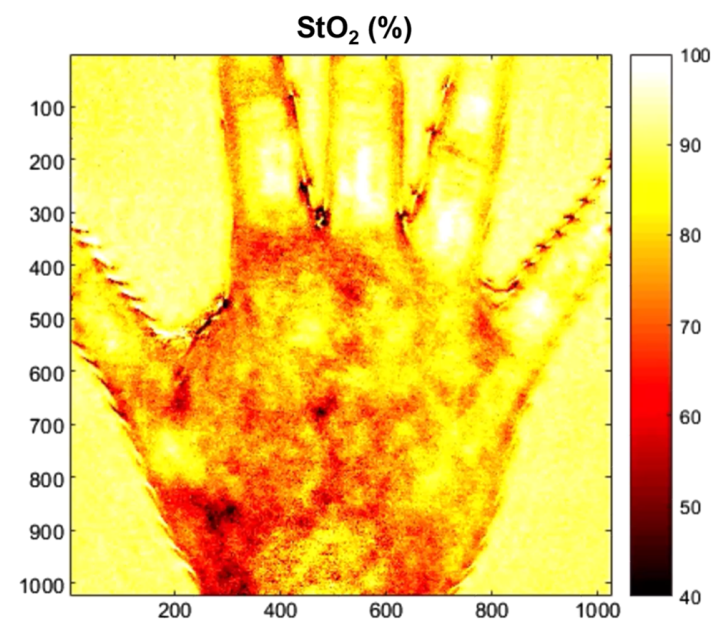

Fig. 6 Video of the arm-cuff occlusion (Video 1, MP4, 74.3 MB [URL: https://doi.org/10.1117/1.JBO.24.7.071610.1]).

\section{Discussion}

In this work, we present the application of a method, spatiotemporal modulation of light, to perform real-time (100 fps), widefield $\left(13 \times 13 \mathrm{~cm}^{2}\right)$, and quantitative imaging of blood parameters in vivo. This method relies on the modulation of light sources in time to encode wavelengths and on the modulation of the projection of these sources in space to extract the optical properties of living tissues. Combined together, these spatiotemporal modulations allow to gather multispectral quantitative optical information in real time (here at $100 \mathrm{fps}$ and postprocessed). Altogether the method enables to provide healthcare professionals with interpretable information that can be used in real time to guide decision making in challenging clinical situations, such as surgery.

Compared to previous work, this study augments the capacity for performing multispectral diffuse optical imaging quantitatively at a high frame rate and over a large field-of-view. For instance, sequential acquisition of two wavelengths using both SSOP and laser speckle imaging acquisition methods led to $16 \mathrm{fps}$ imaging of simultaneous blood parameters and perfusion imaging over a wide field-of-view $\left(15 \times 15 \mathrm{~cm}^{2}\right) .{ }^{12}$ In more recent work, compressed sensing was used along with square patterns projections and phase shifting leading to $12.5 \mathrm{fps}$ imaging of blood parameters over a smaller field-of-view $\left(3.5 \times 3.5 \mathrm{~cm}^{2}\right) .^{13}$

However, this work comes with limitations. First, in this implementation, the SSOP method results in slight image degradation ${ }^{9-11}$ and the 3-D profile of the sample is not measured, which can cause errors in extracting optical properties onto complex, non-flat objects ( $\sim 10 \%$ error per $\mathrm{cm}$ for absorption) ${ }^{26}$ Second, noise in oxygenation data, mainly caused by the poor quantum efficiency of the camera at $860 \mathrm{~nm}$, as well as slight synchronization errors between the camera and the sources, resulted in the impossibility to observe fine variations such as the pulsatile nature of blood flow. Improvements in the imaging system will include stronger illumination at $860 \mathrm{~nm}$ and a custom light source for optimal camera-source synchronization to allow the observation of finer variations of oxygenation. Third, currently images are acquired in real time but postprocessed following acquisition. We are currently working on a hardware and software solutions enabling both real-time acquisition and real-time processing, making this technology deployable for real-time visualization of the surgical field. ${ }^{24,27,28}$ Finally, while the concept can be expanded to virtually many more wavelengths, there is a significant instrumental challenges in acquiring properly all wavelengths with a good enough SNR, and within the limitations of the spectral quantum efficiency and the dynamic range of the camera. For instance, in this work, the 860-nm optical properties, necessary to optimally separate oxy- and deoxyhemoglobin, shows significantly more noise compared with $665 \mathrm{~nm}$ due to the decrease of the spectral quantum efficiency of silicon-based camera technology. While manageable for a few wavelengths, this certainly limits the acquisition of a large number of wavelengths concurrently.

One advantage of this method though is its capacity to work in real time. Current CMOS technology offers cameras at a very reasonable price permitting good resolution and dynamic range imaging up to $500 \mathrm{fps}$. Our GP-GPU implementation of the temporal DFT in CUDA permits processing down to $18 \mu \mathrm{s}$ per wavelength at a resolution of $512 \times 512$ pixels. ${ }^{20}$ However, the rolling window processing approach comes at the cost of a short delay between the acquired scene and the displayed 
information (here 1/10th of a second, i.e., $100 \mathrm{~ms}$ ). One potential solution to decrease this delay is to increase the acquisition rate (500 fps for instance) but it requires to increase the light fluence from the sources to maintain proper SNR and prevent noise in the images.

Finally, current state-of-the-art processing methods in the spatial frequency domain include optimized LUTs (down to $18 \mathrm{~ms}$ for $572 \times 672$ pixels), ${ }^{24}$ as well as deep learning and machine learning methods (down to $200 \mathrm{~ms}$ for $696 \times 520$ pixels), ${ }^{28,29}$ and soon, faster methods that will enable real-time processing and visualization of data will certainly be developed. Efforts are ongoing to combine rapid processing of SSOP data, with spatiotemporal modulation of light, onto a clinically compatible device to test and validate the capacity for diffuse optical imaging to provide relevant data in real time during concrete clinical applications.

\section{Conclusion}

In this work, we presented an approach for real-time, wide-field, and quantitative imaging of physiologically and clinically relevant data onto living tissues. Spatiotemporal modulation of light enables true real-time quantitative multispectral imaging opening unprecedented possibilities for translating such technology to clinically relevant applications where time is constrained, such as surgery. This study contributes to the translation of diffuse optical imaging methods to relevant clinical applications.

\section{Disclosures}

B.J.T. discloses patents in SFDI technology that are assigned to the Regents of the University of California. These patents have been licensed to Modulated Imaging (MI), Inc.; B.J.T. is a cofounder of MI. All other authors declare that there are no conflicts of interest related to this article.

\section{Acknowledgments}

The authors would like to thank Martin van der Mark for his inspiring presentation related to temporal modulation of wavelengths at the "Lasers in Medicine and Biology" Gordon conference in 2010. This work was supported by the European Research Council (ERC, award\# 715737, S.G.) and the University of Strasbourg IdEx (S.G.).

\section{References}

1. A. E. Cerussi et al., "Frequent optical imaging during breast cancer neoadjuvant chemotherapy reveals dynamic tumor physiology in an individual patient," Acad. Radiol. 17(8), 1031-1039 (2010).

2. J. M. Cochran et al., "Tissue oxygen saturation predicts response to breast cancer neoadjuvant chemotherapy within 10 days of treatment," J. Biomed. Opt. 24(2), 021202 (2018).

3. A. Yafi et al., "Quantitative skin assessment using spatial frequency domain imaging (SFDI) in patients with or at high risk for pressure ulcers," Lasers Surg. Med. 49(9), 827-834 (2017).

4. C. Weinkauf et al., "Near-instant noninvasive optical imaging of tissue perfusion for vascular assessment," J. Vasc. Surg. 69(2), 555-562 (2018).

5. B. W. Pogue et al., "Fluorescence-guided surgery and intervention: an AAPM emerging technology blue paper," Med. Phys. 45(6), 2681-2688 (2018).

6. N. Dognitz and G. Wagnieres, "Determination of tissue optical properties by steady-state spatial frequency-domain reflectometry," Lasers Med. Sci. 13(1), 55-65 (1998).
7. D. J. Cuccia et al., "Quantitation and mapping of tissue optical properties using modulated imaging," J. Biomed. Opt. 14(2), 024012 (2009).

8. J. P. Angelo et al., "Review of structured light in diffuse optical imaging," J. Biomed. Opt. 24(7), 071602 (2018).

9. J. Vervandier and S. Gioux, "Single snapshot imaging of optical properties," Biomed. Opt. Express 4(12), 2938-2944 (2013).

10. K. P. Nadeau, A. J. Durkin, and B. J. Tromberg, "Advanced demodulation technique for the extraction of tissue optical properties and structural orientation contrast in the spatial frequency domain," J. Biomed. Opt. 19(5), 056013 (2014).

11. M. van de Giessen, J. P. Angelo, and S. Gioux, "Real-time, profilecorrected single snapshot imaging of optical properties," Biomed. Opt. Express 6(10), 4051-4062 (2015).

12. M. Ghijsen et al., "Quantitative real-time optical imaging of the tissue metabolic rate of oxygen consumption," J. Biomed. Opt. 23(3), 036013 (2018).

13. M. Torabzadeh et al., "Compressed single pixel imaging in the spatial frequency domain,” J. Biomed. Opt. 22(3), 030501 (2017).

14. S. Gioux et al., "First-in-human pilot study of a spatial frequency domain oxygenation imaging system," J. Biomed. Opt. 16(8), 086015 (2011).

15. J. R. Weber et al., "Multispectral imaging of tissue absorption and scattering using spatial frequency domain imaging and a computed-tomography imaging spectrometer," J. Biomed. Opt. 16(1), 011015 (2011).

16. A. Yafi et al., "Postoperative quantitative assessment of reconstructive tissue status in a cutaneous flap model using spatial frequency domain imaging," Plast. Reconstr. Surg. 127(1), 117-130 (2011).

17. M. B. Applegate and D. Roblyer, "High-speed spatial frequency domain imaging with temporally modulated light," J. Biomed. Opt. 22(7), 076019 (2017).

18. R. H. Wilson et al., "High-speed spatial frequency domain imaging of rat cortex detects dynamic optical and physiological properties following cardiac arrest and resuscitation," Neurophotonics 4(4), 045008 (2017).

19. J. O. Smith, Mathematics of the Discrete Fourier Transform (DFT): with Audio Applications, W3K, Stanford, California (2007).

20. E. Aguenounon et al., "Real time hyperspectral imaging using high frame rate video camera and GPGPU processing," in SIGNAL, Nice, France (2018).

21. A. Mazhar et al., "Wavelength optimization for rapid chromophore mapping using spatial frequency domain imaging," J. Biomed. Opt. 15(6), 061716 (2010).

22. F. Ayers et al., "Fabrication and characterization of silicone-based tissue phantoms with tunable optical properties in the visible and near infrared domain," Proc. SPIE 6870, 687007 (2008).

23. T. A. Erickson et al., "Lookup-table method for imaging optical properties with structured illumination beyond the diffusion theory regime," J. Biomed. Opt. 15(3), 036013 (2010).

24. J. Angelo et al., "Ultrafast optical property map generation using lookup tables," J. Biomed. Opt. 21(11), 110501 (2016).

25. G. J. Greening et al., "Characterization of thin poly(dimethylsiloxane)based tissue-simulating phantoms with tunable reduced scattering and absorption coefficients at visible and near-infrared wavelengths," J. Biomed. Opt. 19(11), 115002 (2014).

26. S. Gioux et al., "Three-dimensional surface profile intensity correction for spatially modulated imaging," J. Biomed. Opt. 14(3), 034045 (2009).

27. J. P. Angelo, M. van de Giessen, and S. Gioux, "Real-time endoscopic optical properties imaging," Biomed. Opt. Express 8(11), 5113-5126 (2017).

28. S. Panigrahi and S. Gioux, "Machine learning approach for rapid and accurate estimation of optical properties using spatial frequency domain imaging," J. Biomed. Opt. 24(7), 071606 (2018).

29. Y. Zhao et al., "Deep learning model for ultrafast multifrequency optical property extractions for spatial frequency domain imaging," Opt. Lett. 43(22), 5669-5672 (2018).

Biographies of the authors are not available. 